\title{
No-Show Rates in the Vascular Laboratory: Analysis and Possible Solutions
}

\author{
Bhagwan Satiani, MD, MBA, FACHE, Susan Miller, RVT, and Darshan Patel, BS ${ }^{1}$
}

PURPOSE: To report the consequence of missed appointments ("no-shows") in the noninvasive vascular laboratory of a large teaching hospital and evaluate the effect of one potential solution.

MATERIALS AND METHODS: The financial effect of missed appointments by outpatients during a 9-month period was calculated on the basis of weighted average reimbursement rates for the technical component of a bilateral venous duplex examination. In addition, the effect of an automated telephone reminder system on the no-show rate was studied over a subsequent 17 -month period.

RESULTS: The overall no-show rate for outpatients in the vascular laboratory was $12 \%$, with an average of 7.6 missed appointments per week. This translated to a gross annual revenue loss of $\$ 89,107$ assuming a per-appointment revenue equal to the $\mathbf{2 0 0 5}$ technical component of the reimbursement rate for outpatient bilateral duplex venous ultrasound studies. Of the 8,766 patients offered automated reminder calls, 4,648 $(53 \%)$ agreed to receive the calls. The no-show rate was significantly greater for those patients who chose to receive automated reminder calls $(8.9 \% \mathrm{vs} 5.9 \%, P<.0001)$.

CONCLUSIONS: A 12\% no-show rate offers an opportunity for significant cost savings and improved efficiency in the vascular laboratory. Automated reminder systems did not appear to significantly reduce the no-show rate. Various strategies are outlined to achieve the goal of a 5\% no-show rate, including methods of scheduling, pre-examination notification, and advanced overbooking techniques. Further investigation into these strategies to reduce the no-show rate is needed.

J Vasc Interv Radiol 2009; 20:87-91

MISSED patient appointments have an adverse effect on resource utilization in healthcare services offered by hospitals and physicians. Beyond a certain threshold, missed appointments lead to lost revenue, inefficient scheduling, and underutilized personnel while simultaneously affecting timely access to healthcare (1). A July 2005

From the Peripheral Vascular Laboratory, Ross Heart Hospital (B.S., S.M.) and the Division of Vascular Surgery (B.S., D.P.), Ohio State University School of Medicine, N333 Means Hall, 1654 Upham Dr, Columbus, OH 43210. Received March 4, 2008; final revision received September 15, 2008; accepted September 26, 2008. Address correspondence to B.S.; E-mail: Bhagwan.satiani@osumc.edu

${ }^{1}$ Current address: University of Pennsylvania School of Medicine, Philadelphia, Pennsylvania.

None of the authors have identified a conflict of interest.

(C) SIR, 2009

DOI: 10.1016/j.jvir.2008.09.027 survey of 217 clinical and surgical medical groups by the Medical Management Group Association (2) found that $88.9 \%$ of respondents track patient no-show rates and use patient appointment reminders. The results of the survey indicate that, at least in the clinical setting, patient no-shows are recognized as a drag on operating efficiency and optimal patient care. Previous studies $(3,4)$ have addressed the financial benefits of reducing no-show rates in the clinical setting by using strategies such as telephone and mail reminders.

Although previous reports have addressed the subject of missed appointments in the clinical setting, the effect of no-shows on the noninvasive vascular laboratory setting has not been adequately studied. Given the importance of prompt and timely laboratory testing to overall patient care, no-show rates in the laboratory setting also merit attention. Vascular laboratories in particular are conducive to detailed financial and operating efficiency analyses because they are relatively small, task-specific settings with predictable appointment duration times (5). The purpose of this retrospective study was to examine the financial and scheduling effect of no-shows on the vascular laboratory setting. In addition, we review various methods used to improve patient compliance as well as the literature on "overbooking" in order to suggest lessons learned in non-healthcare industries.

\section{MATERIALS AND METHODS}

All data for the no-show part of the study were collected in the peripheral vascular laboratory between January 2005 and September 2005. The vascular laboratory did not use patient appointment reminders during the initial phase of the study. Only outpatient procedures scheduled before the day 


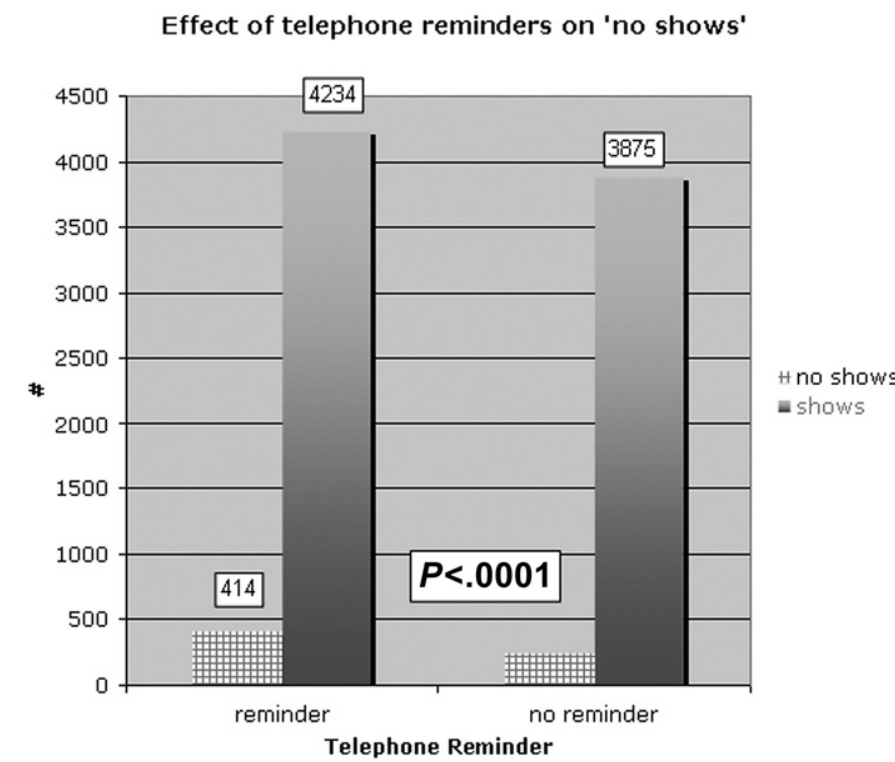

Figure 1. Chart shows no-show rates for patients who chose to receive telephone reminders and those who did not.

of appointment were included in the study. Patients who did not show up for their scheduled vascular testing appointment were labeled as no-shows. The study protocol was part of a larger study on portable ultrasonography (US) testing that was reviewed and approved by the institutional review board.

Vascular laboratory schedule data for an uninterrupted 5-day work week (Monday to Friday) was selected from each of the 9 months of the study period. The schedule data were used to tabulate the total number of outpatient appointments included in the study as well as the total number of no-shows within this outpatient population. Noshow appointments were marked "NS" or "cancelled" on daily schedules by vascular laboratory staff, facilitating accurate data collection. An overall noshow rate for the vascular laboratory was then estimated by using this data. During this time period, the lag time between scheduling the test and the first available appointment ranged from 15 to 30 days.

Recent Medical Management Group Association data indicating a no-show rate of $5 \%$ were used as a reasonable target to achieve in the vascular laboratory. To calculate the incremental revenue associated with the potential additional laboratory studies, we used payment for an outpatient bilateral duplex venous US study, the most common outpatient procedure per- formed in the vascular laboratory. The technical reimbursement for 2005 rates for government payers (Medicare, Medicaid, and state prison system) and the five largest commercial payers were recorded along with the frequency (in percentage) of each payer. A blended rate was then calculated for all payers on the basis of the percentage of total contribution to the vascular laboratory.

This per-study reimbursement rate was multiplied by the annual incremental appointments figure to calculate incremental annual revenue due to the reduced no-show rate.

An automated patient reminder phone system was used starting in January 2006 and was offered to all patients registering to schedule an outpatient vascular test. Patients were asked at the time of registration whether they would like a telephone reminder. The telephone message was as follows: "This is OSU medical center calling for first name to remind you of your appointment on XXX - press 1 to confirm, press 2 to cancel." The financial cost of the reduced no-show rate was based on the annual expenses associated with an automated patient reminder call system (Audiocare, Berwyn, Pennsylvania). The annual cost of the system for the vascular laboratory was provided to us by the hospital's finance department. This cost was deducted from the incremental reve- nue to derive the total financial benefit of reducing the vascular laboratory no-show rate.

The effect of the reminder phone message system during a 17-month period from July 1st, 2006, to November 30th, 2007, was analyzed. A total of 8,938 outpatients were scheduled for vascular laboratory tests, of whom 4,648 patients $(53 \%)$ agreed to receive a reminder phone message 3-4 days before their appointment; 4,118 patients $(47 \%)$ chose not to receive the phone call. Of the 4,648 who received a phone reminder, 414 did not show for their appointment $(8.9 \%)$ compared to 243 of 4,118 (5.9\%) who chose not to receive a reminder phone message $(P<.0001)($ Fig 1$)$.

\section{RESULTS}

The vascular laboratory scheduled a total of 558 outpatient procedures in advance of the appointment time during the first phase of the study period, of which $68(12 \%)$ were classified as noshows. On a weekly basis, the vascular laboratory recorded an average of 7.6 missed appointments. In financial terms, these missed appointments represented a gross annual loss of $\$ 89,107$ assuming a per-appointment revenue equal to the 2005 technical component of the blended reimbursement rate for outpatient bilateral duplex venous studies. Reducing the no-show rate to the 5\% average reported by the Medical Management Group Association would increase vascular laboratory revenue by $\$ 51,769.00$ (\$89,107.00-\$37,338.00) (Fig 2). 
These missed appointments also create a drag on scheduling efficiency, increasing the wait time for outpatients.

Our medical center currently uses an automated patient reminder call system throughout much of the hospital system. The estimated fixed annual costs of the system, including support and maintenance, are approximately $\$ 10,000$. However, because the annual maintenance fee is shared across all groups within the hospital using the system on the basis of the actual percentage of appointments and/or calls made, the fixed cost to the vascular laboratory is estimated at only $\$ 60.89$ per year. Adding to this the variable costs of both local and long-distance calls (\$231.46) brings the total annual costs of the system to $\$ 292.35$ for the vascular laboratory. This is a relatively small sum compared to the incremental revenue figures discussed earlier.

\section{DISCUSSION}

Missed appointments (bookings) are a ubiquitous problem common to many industries such as airlines, railways, hotels, and cruise lines $(6,7)$. For outpatient facilities and clinics, noshow appointments clearly represent a lost revenue opportunity in addition to inefficiencies from idle time and the increased wait times for new appointments.

The range of no-show patients varies from $5 \%$ to $39 \%$ according to the type of facility or practice (8-10). Almost all reports in this regard focus on outpatient clinics in various specialties, including family practice, pediatrics, and mental health. However, there are almost no studies on the scope of the problem in the area of diagnostic procedures. Vascular US is a very specialized area, with highly paid technologists, expensive fixed costs associated with advanced technology, and, in general, the inability to freely substitute another patient needing a test on short notice unless the facility has inpatients. A $12 \%$ no-show rate in our outpatient population resulted in an estimated annual gross loss of $\$ 89,107$ under the assumptions listed in our study. A no-show rate of $5 \%$ would reduce the loss and additional revenue to the hospital of $\$ 51,769$ annually. Before instituting a method for dealing with no-shows, the laboratory must identify what the no- show rate is and the financial effect on the vascular laboratory. The average revenue for the procedure is multiplied by the number of no-show appointments. As an example, let us assume that a free-standing vascular laboratory that collects global fees, the total weighted reimbursement (calculated by the average of the top five payers weighted by the actual percentage of the payers in the practice) is $\$ 400$. Also assume that the vascular laboratory does approximately 10,000 tests each year and the no-show rate is $10 \%$. Then, $10,000 \times 0.10=1,000$ no-show appointments $\times \$ 400=\$ 400,000$. Let us also assume that the vascular laboratory has a target no-show rate of $5 \%$. Compared with a $10 \%$ no-show rate, the improvement in revenues is $\$ 200,000$.

Some authors have attempted to use six sigma tools (Motorola Inc, Schaumburg, Illinois) to identify the root causes for patients not showing for their appointment (11). The root cause may vary from parking concerns, inconvenient time of the day, costs, and child care needs to long expected wait times (12). Rose and Chung (4) reported a no-show rate of $17 \%$ in a large group practice and found insurance to be the strongest predictor, with Medicaid patients being three times as likely and private pay patients twice as likely to be noshows. However, the root cause in this population may be transportation. Once the manager understands the chief root causes, an attempt is made to address these rather than accept a certain no-show rate and compensate by overbooking. In a study of 200 patients who failed to attend a clinic, Collins and colleagues (9) determined that the major reason for not showing was the patient's opinion about difficulty in getting to the hospital. In addition, previous nonattendance was found to be a strong predictor of future nonattendance.

There are three common methods of scheduling outpatients for physician offices and vascular laboratories. The proportional method relies on separating appointments by the length of time it takes for the visit or test. Most vascular laboratories use this method because average times for common tests have a small standard deviation. Wave scheduling involves having several patients arrive at the same time followed by another "wave" in the next hour. Vascular laboratories are not likely to use this method as the only technique to deal with a busy schedule but often use it in combination with the proportional method (modified wave scheduling). The third or clinic method entails scheduling patients with similar diseases in the same time frame.

Vascular laboratory managers are somewhat limited in applying the solutions that may be considered by medical office managers. For instance, charging for missed appointments is generally not an option because the patient is not well known to the testing facility and it risks antagonizing the referring physician. A possible scenario that may mitigate against the negative effect of no-shows in an office practice is that of promoting patients added to the schedule either because they are urgent or referral patterns. Whereas outpatient primary care clinics may encourage these "add-ons," a vascular laboratory is not suited for unscheduled patients. For those highvolume vascular laboratories with substantial in-patient facilities, add-on tests may replace no-shows as a book of business that can reduce loss of revenue. All medical facilities, in general, have two options that are realistically available to address no-shows. They are (a) strategies to minimize noshows such as pre-examination notification (11) and self-scheduling for patients and $(b)$ overbooking.

Reminder cards are a popular method of reminding patients about their upcoming appointment (10). Another widespread method is having office staff make phone calls to patients to remind them of their upcoming appointment. In small vascular laboratories, the staff may have enough time at the end of the day to make the reminder calls. In large practices or busy vascular laboratories, however, phone calls by staff may just not be a viable option. Conversely, the advantage of automatic phone reminder systems is that instead of the end of the day, they can be programmed to call at a time of choice and document the completion of the call.

For high-volume vascular laboratories based in a hospital like ours, the automated patient reminder call system was considered to be an efficient way to achieve this reduction in no-shows. The fixed costs of such a system could be spread across multiple cost centers 
while giving each division the potential to reduce no-show rates. Most telephone reminder systems are fairly costeffective because they generally require no special interface and, therefore, no postpurchase maintenance expenses. The largest companies are Televox (Mobile, Alabama; www.Televox.com), Julysoft (Tucson, Arizona; www.julysoft. com), Phonetree (Winston-Salem, North Carolina; www.phonetree.com), and MedVoice (Scottsdale, Arizona; www. medvoice.com). Audiocare (http:// www.audiocare.com) is a module within IDX Systems (a software company owned by GE, Fairfield, Connecticut), a patient scheduling system through which generic and Health Insurance Portability and Accountability Act-compliant reminder calls can be scheduled. It gives the recipient the option to accept the appointment, cancel the appointment, or leave a message.

Previous studies evaluating the effectiveness of telephone reminders to improve patient compliance at outpatient clinics have yielded mixed results. Danoff and Kemper (8) reported no difference between patients who received telephone reminders and those who did not in an urban pediatric clinic. In a prospective, randomized study of an urban family practice population, Hashim et al (3) showed a significantly larger number of no-shows $(26 \%)$ in the group who received telephone reminders compared to the group who did not $(19 \%)(P<.006)$. An additional advantage noted in the telephoned group was a higher rate of cancellation, which allowed the use of those slots for other patients. Our data show that about half of our patients (53\%) signed on to accept the offer of an automated phone call reminder. However, the no-show rate in this group $(8.9 \%)$ was significantly greater than the $5.9 \%$ no-show rate for patients who chose not to receive the phone message. One could argue that the latter group was a more compliant group to begin with in the sense that they did not see the need for a reminder. The fact remains that the $8.9 \%$ no-show rate for those receiving a reminder is high and even though the cost of the reminder system is reasonable, this method to reduce the noshow rate does not appear to be very successful.

With the number of internet users rising, the option for patients to sched- ule an appointment that fits their schedule seems intuitively to offer advantages over the current system. However, for various reasons including fear of patient confidentiality and cost, self-scheduling has not become widespread.

Significant improvement in profitability has been reported by adopting revenue management in industries such as airlines, auto rental, cruise lines, passenger railways, broadcasting, hotels, and restaurants. Revenue management is simply a process of "selling the right type of capacity to the right customer, at the right price, and at the right time" (6). Forecasting demand and overbooking are two pieces that have been heavily researched as part of revenue management. Comparison between the healthcare sector and the transportation sector may be worth reviewing. The airlines use two variations in implementing revenue management methodology: manipulation of the release of inventory (seats) at fixed prices or manipulation of prices at varying times (6). In the transportation sector, clients arrive at one time whereas in healthcare the appointments (and no-shows) are spread out over time. In addition, the inventory (seat) is relatively fixed and prices are variable depending on pricing structure, favoring the "early bird" (customers who call earliest). A "seat mix" problem (where airlines try to maximize revenue by limiting sales to low-revenue passengers and yet make sure all seats are filled) is not tenable in healthcare. Because most hospitals and physician offices are severely restricted in raising prices, the only reasonable option is to use resources more efficiently. Some office practices decline to reschedule chronic no-shows or demand a small fee after two or three cancellations, and some industries penalize no-shows (a charge to reschedule a missed airline trip). However, to not upset referring physicians, no vascular laboratory to our knowledge has put financial penalties or refused to reschedule chronic noshows. It is clear that revenue management in the form practiced at airlines, for instance, to enhance revenues will not be a realistic option in solving the problem in this scenario.

Overbooking is a component of revenue management that may lend itself to being adapted for healthcare. Some office practices resort to "double booking" in order to avoid wasting resources, including valuable physician time. This is simply a tactic to deal with the certainty that some patients will not show for their appointment by scheduling more patients than the physician has time to see during those hours. It does not address root causes of not showing for appointments but does improve patient access and physician and staff productivity. A disadvantage of random overbooking without analyzing no-show data may lead to prolonged wait times with associated patient dissatisfaction, unhappy staff, and overtime costs for the practice or hospital. As LaGanga and Lawrence (13) point out, balancing the tradeoffs of serving additional patients and improving productivity versus prolonging patient wait times, occasional staff fatigue, and, possibly, overtime costs is the key. The authors demonstrated that overbooking in healthcare clinics can have a positive effect by increasing patient access and improving productivity (13). However, overbooking can result in increased waiting times for patients and staff overtime expenses. On the basis of simulation experiments, regression analysis, and sensitivity experiments, LaGanga and Lawrence (13) concluded that, in general, overbooking is more advantageous when a clinic or facility serves large numbers of patients, no-show rates are high, and service variability is lower. Their recommendations may be applicable to large, busy vascular laboratories with a high no-show rate.

Healthcare systems and physician offices must monitor no-show patient statistics and put policies in place to minimize inefficiencies and improve revenue opportunities. Vascular laboratories serving both inpatients and outpatients with a large in-patient population generally have patients waiting to get urgent tests performed. A no-show rate of $12 \%$ probably is of very little practical importance because the empty slots are easily filled every day with inpatient requests. Chaos may still result because of the delay in transporting inpatients to the laboratory after laboratory personnel have waited for the no-show outpatient and then decided to fill the empty slot. However, vascular laboratories serving solely outpatients or low-census inpatient facilities with a high no-show rate should consider an overbooking model 
such as the stochastic mathematical overbooking model proposed by Kim and Giachetti (14) rather than a "naïve" overbooking model. A stochastic model involves a nondeterministic, more "random" process and considers the probability distribution of no-shows as well as walk-in patients. In addition, it considers the cost structure so as to maximize expected revenue. Through sensitivity analysis, Kim and Giachetti (14) have demonstrated improved total profits in high and stochastic no-show rate situations. The model importantly does assume a low variability in patient service times. Fortunately, the variance in service times for the most common vascular tests is small (15).

There are other common-sense measures to reduce no-show rates, such as establishing a separate cancellation phone line, encouraging people to be good citizens, having a waiting list similar to "stand by" seating in the airlines industry, monitoring repeat no-shows, and having a patient discharge policy for repeat and egregious violators.

Several limitations of our study deserve mention. The present study was conducted at a large university hospital in the United States, and our findings may not be applicable to other countries or dissimilar institutions. Our study was not a prospective, randomized study and does not address the root causes that we have mentioned. A possible limitation of the telephone reminder is that patients may not have remembered that the reminder was automated. So, when they heard the "click" from the autodialer switching over to the message, they may have hung up before hearing anything because they assumed it was a telemarketing call. Furthermore, the lag time between scheduling the test and the date of the test may have resulted in patients failing to remember their appointments.

In summary, a $12 \%$ no-show rate for outpatients scheduled for noninvasive vascular testing offers an opportunity for significant cost savings and improved efficiency in the vascular laboratory. Automated reminder systems, reported to be useful in outpatient clinics, did not appear to significantly reduce the no-show rate. Various strategies are discussed to achieve a no-show goal rate of $5 \%$, including methods of scheduling, pre-examination notification, and advanced overbooking techniques. Lessons learned from our study may be applicable to other imaging procedures as well. Further investigation into these strategies to reduce the no-show rate is needed.

Acknowledgment: We thank Linda Alpers for information and assistance with data regarding telephone reminders.

\section{References}

1. Macharia WM, Leon G, Rowe BH, Stephenson BJ, Haynes RB. An overview of interventions to improve compliance with appointment keeping for medical services. JAMA 1992; 267: 1813-1817.

2. Performances and practices of successful medical groups. Englewood, Colorado: MGMA, 2005

3. Hashim MJ, Franks P, Fiscella K. Effectiveness of telephone reminders in improving rate of appointments kept at an outpatient clinic: a randomized controlled trial. J Am Board Fam Pract 2001; 14:193-196.

4. Rose MS, Chung MK. On with the show. MGMA Connexion 2003; 8:54-57.

5. Fillinger M. Cost-effective use of the noninvasive vascular laboratory: potential trends related to increased eco- nomic pressures. Semin Vasc Surg 1997; 10:98-105.

6. Smith BC, Leimkuhler JF, Darrow RM. Yield management at American Airlines. Interfaces 1992; 22:8-31.

7. Bitran G, Caldentey R. Commisioned paper: an overview of pricing models for revenue management. Manufacturing Serv Operations Management 2003; 5:203-229.

8. Danoff NL, Kemper KJ. Does excluding patients without telephones affect the results of telephone reminder studies? West J Med 1993; 158:44-46.

9. Collins J, Santamaria N, Clayton L. Why outpatients fail to attend their scheduled appointments: a prospective comparison of differences between attenders and non-attenders. Aust Health Rev 2003; 26:52-63.

10. Rust CT, Gallups NH, Clark WS, Jones DS, Wilcox WD. Patient appointment failures in pediatric resident continuity clinics. Arch Pediatr Adolesc Med 1995; 149:693-695.

11. Yarbro D. Solving the dilemma created by the no-show patient. Six Sigma Healthcare. http://healthcare.isixsigma.com/ library/content/c040204a.asp?actio=print. Accessed February 2, 2008.

12. Sharp DJ, Hamilton W. Non-attendance at general practices and out-patient clinics: local systems are needed to address local problems. Br Med J 2001; 323:1081-1082.

13. LaGanga LR, Lawrence SR. Clinic overbooking to improve patient access and increase provider productivity. Decis Sci 2007; 38:251-276.

14. Kim S, Giachetti RE. A stochastic mathematical appointment overbooking model for healthcare providers to improve profits. IEEE Trans Systems Man Cybernetics. Part A: Systems and Humans, 2006; 36:1211-1219.

15. Patel D, Satiani B, Mong R, Baetz L, Spiezio K. Appropriate resource utilization in portable noninvasive vascular studies: the role of disruptive technology. J Vasc Ultrasound 2006; 30:35-38. 